\title{
МЕТОДИКА ОЦЕНКИ ДОВЕРИТЕЛЬНОГО ИНТЕРВАЛА ПРИ ОПРЕДЕЛЕНИИ МИКРОКОМПОНЕНТА РЕНТГЕНО-ФЛУОРЕСЦЕНТНЫМ МЕТОДОМ
}

(Представил О. Эйзен)

Рентгено-флуоресцентный метод анализа можно отнести к числу самых перспективных методов неорганического анализа. Он позволяет определить большинство химических элементов. При определении микрокомпонентов этим методом особое внимание следует обратить на доверительный интервал результата анализа. Цель настоящей работы разработка методики оценки доверительного интервала определения концентрации микрокомпонента.

При анализе рентгено-флуоресцентным методом необходимо учитывать эффект матрицы [1]. Для решения этой задачи предложено пользоваться некогерентно рассеянным на пробе характеристическим излучением анода рентгеновской трубки $\left[^{2}\right]$. Тогда концентрацию $C$ определяемого элемента можно вычислить по уравнению

$$
C=K I_{\text {л }} / I_{\text {В }}
$$

где $I_{л}$ - «чистая» (без фона) интенсивность аналитической линии определяемого элемента; $I_{\text {н }}$ - интенсивность некогерентно рассеянного излучения; $K-$ коэффициент пропорциональности. Следует иметь в виду, что на излучение аналитической линии накладывается т. н. фоновое излучение, которым нельзя пренебречь в случае низких содержаний определяемого элемента. Тогда «чистая» интенсивность аналитической линии это - разность измеряемой интенсивности аналитической линии $i_{\text {л }}$ и интенсивности фона $i_{\text {ф }}$

$$
I_{\pi}=i_{\pi}-i_{\Phi}
$$

Интенсивность фонового излучения складывается из нескольких компонентов; в числе которых превалирует некогерентно рассеянное на пробе первичное рентгеновское излучение. Интенсивность фона хорошо аппроксимируется уравнением [3]

$$
i_{\Phi}=a I_{\mathrm{H}}+b .
$$

Коэффициенты $a$ и $b$ этой линейной зависимости определяются по холостым пробам различного химического состава. Коэффициент пропорциональности $K$ (1) устанавливается по стандартным образцам [ $\left.{ }^{4}\right]$

$$
\mathrm{K}=\sum_{m=1}^{n} X_{m}^{*} C_{m}^{*} / \sum_{m=1}^{n}\left(X_{m}^{*}\right)^{2},
$$

где $n$ - количество использованных стандартных образцов; $C_{m}^{*}-$ концентрация определяемого элемента в стандартном образце $m$; $X_{m}^{*}$ - отношение интенсивности «чистой» аналитической линии к интенсивности некогерентно рассеянного излучения стандартного образца 


$$
X_{m}^{*}=I_{\mathrm{\pi}, m}^{*} / I_{\mathrm{H}, m}^{*} \text {. }
$$

Для оценки доверительного интервала определяемой концентрации $C$ (1) используем закон распространения погрешностей [ $\left.{ }^{4}\right]$

$$
E_{C}^{2}=E_{I_{\pi}}^{2}+E_{K}^{2}+E_{I_{\mathrm{H}}}^{2},
$$

где $E_{I_{л}}, E_{K}$ и $E_{I_{\text {н }}}$ - относителыные среднеквадратичные погрешности $I_{\text {л }}(2), K(4)$ и $I_{\text {н }}$ соответственно.

Рассмотрим слагаемые уравнения (6). Первое из них,

$$
E_{I_{л}}=\Delta I_{\text {л }} / I_{\text {л }}=K \Delta I_{\text {л }} / C I_{\text {н, }}
$$

содержит $\Delta I_{\text {л }}$, представляющую собой среднеквадратичную погрешность

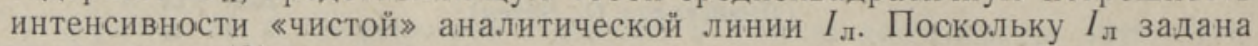
уравнением (2), то по закону распространения погрешностей

$$
\Delta I_{\pi}^{2}=\Delta i_{\Phi}^{2}+\Delta i_{\pi}^{2} .
$$

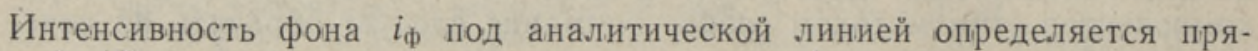
мой (3); соответствующие параметры $a$ и $b$ вычисляются методом наименьших квадратов [ $\left.{ }^{4}\right]$ с использованием значений $i_{\Phi}$ и $I_{\mathrm{H}}$, измеренных при помощи ряда холостых проб различного химического состава. Вследствие неизбежной погрешности определения коэффициентов $a$ и $b$ необходимо рассматривать $i_{\Phi}$ (3) как случайную величину. Довери-

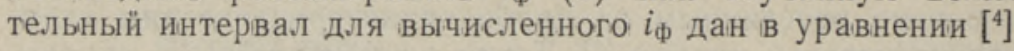

$$
S_{\Phi}^{2}=S_{0}^{2}+S_{a}^{2}\left(I_{\mathrm{H}}-\bar{I}_{\mathrm{H}}\right)^{2}
$$

где $S_{0}$ - среднеквадратичная погрешность регрессии (3); $S_{a}$ - среднеквадратичная погрешность коэффициента $a ; \bar{I}_{\text {н }}-$ среднее значение аргумента $I_{\text {н }}$ при определении коэффициентов $a$ и $b$ по холостым пробам. Однако полуширину коридора попрешностей $S_{\Phi}(9)$ нельзя принимать за $\Delta i_{\phi}$, так как при определении микрокомпонента в анализируемой пробе величину $I_{\text {н }}$ следует рассматривать также как случайную с относительной погрешностью $E_{I_{\text {н }}}$. Таким путем вводится дополнительная погрешность

$$
\Delta I_{\mathrm{H}}=I_{\mathrm{H}} E_{I_{\mathrm{H}}}{ }^{\prime}
$$

Поскольку $S_{\Phi}(9)$ и $\Delta I_{\text {н }}(10)$ независимы друг от друга, то по закону распространения попрешностей

$$
\Delta i_{\Phi}^{2}=S_{\Phi}^{2}+a^{2}\left(\Delta I_{\mathrm{H}}\right)^{2} .
$$

Кроме $\Delta i_{\Phi}$ выражение для $\Delta I_{\text {л }}(8)$ содержит также

$$
\Delta i_{\pi}=\left[(C / K+a) I_{\mathrm{H}}+b\right] E_{i_{\pi}} .
$$

Уравнение (12) получено в результате умножения относительной попрешности $i_{л}\left(E_{i_{л}}\right)$ на $i_{л}$; последняя величина легко вычисляется по уравнениям (1)-(3).

По уравнениям $(9)-(12)$ можно вычислить $\Delta I_{\text {л }}(8)$. Следовательно, это позволяет считать решенным вопрос об оценке первого слагаемого $E_{I_{л}}(7)$ в полиноме (6). Следующий член в (6) $E_{K}$ представляет собой относительную среднеквадратичную погрешность коэффициента $K$ (4). Для линейной зависимости, проходящей через начало координат, среднеквадратичная погрешность углового коэффициента $K$ вычисляется уравнением [ $\left.{ }^{4}\right]$ 


$$
E_{K}^{2}=\left(\sum_{m=1}^{n} C_{m}^{*}-K \sum_{m=1}^{n} X_{m}^{*} C_{m}^{*}\right) /(n-1) \sum_{m=1}^{n}\left(X_{m}^{*}\right)^{2}
$$

Обозначения те же, что и в уравнении (4).

Наконец, осталось последнее слагаемое в полиноме (6). Это $E_{I_{\mathrm{H}}}-$ относительная среднеквадратичная погрешность интенсивности некогерентно рассеянного излучения. Единственная возможность для оценки $E_{I_{\mathrm{H}}}-$ многократное измерение $I_{\mathrm{H}}$ в одних и тех же условиях. По этим данным легко оценить $E_{I_{\text {H }}}$, применяя стандартные процедуры математической статистики [ [ $]$.

Из приведенных выше уравнений следует, что доверительный интервал определяемой по (1) концентрации $C$ зависит от двух прупп факторов. Первая из них - среднеквадратичные погрешности $E_{i_{\pi}}, E_{I_{\mathrm{H}}}, E_{K}$ и $S_{a}$. Вторую группу факторов представляют значения $C, K, I_{\mathrm{H}}, a$ и $\bar{I}_{\mathrm{H}}$. Однако при определении данного микрокомпонента большинство указанных параметров задано условиями эксперимента: $K, \bar{I}_{\mathrm{H}}, a, S_{a}, E_{K}, E_{i_{\text {I }}}$ и $E_{I_{\mathrm{H}}}$. С каждой анализируемой пробой варьируются лишь два параметра: концентрация микрокомпонента $C$ и интенсивность некогерентно рассеянного излучения $I_{\mathrm{H}}$, отражающего свойства матрицы пробы. Поэтому доверительный интервал для $C$ фактически является функцией двух переменных ( $C$ и $I_{\text {н }}$ ) при фиксированных значениях всех остальных перечисленных параметров. Задавая для $I_{\text {н }}$ некоторые фиксированные значения, нередко встречающиеся у анализируемых проб, по вышеизложенной методике можно вычислить семейство кривых $E_{C}=\left.F(C)\right|_{I_{\mathrm{H}}}$. На рисунке приведены кривые

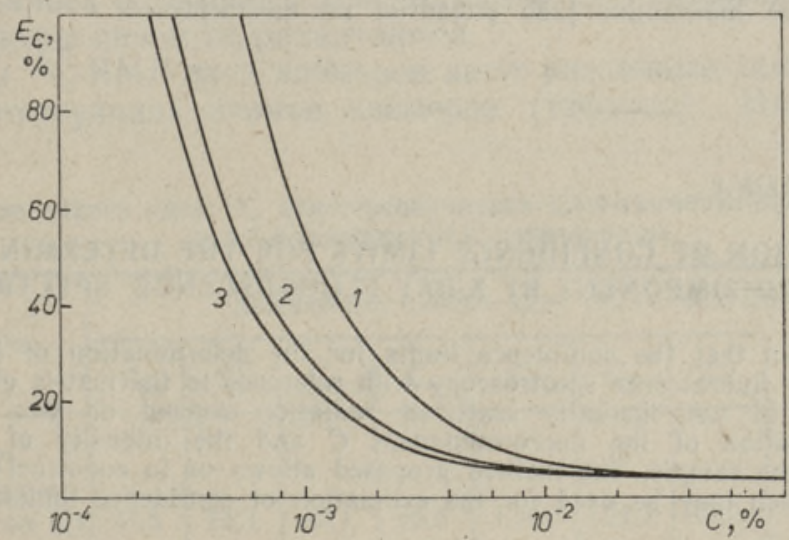

Зависимость доверительного интервала $E_{C}$ от концентрации микрокомпонента $C$ при интенсивности некогегрентно рассеянного на пробе излучения 2000 (1), 4000 (2) и 6000 uмn $/ c$ (3).

$E_{C}=\left.F(C)\right|_{I_{\mathrm{H}}}$ для одного микрокомпонента с параметрами $E_{i_{\pi}}=0,02$, $E_{I_{\mathrm{H}}}=0,02, E_{K}=0,005, K=0,1182, \bar{I}_{\mathrm{H}}=4475, a=0,0438, S_{a}=0,0013$. Указанные значения параметров получили, используя выпускаемый в СССР рентгено-флуоресцентный анализатор АРФ-6. Следует отметить, что анализируемые нами на этом приборе образцы имеют интенсивность некогерентно рассеянного излучения $I_{\mathrm{H}}=2000-6000$ uмn/c. Относительный доверителыный интервал $E_{C}$ расширяется с уменьшением опреде- 
ляемой концентрации $C$ (рисунок). Если предельно допустимая ширина доверительного интервала составляет $50 \%$ соответствующего значения $C$, то по данным, приведенным на рисунке, минималыную определяемую концентрацию можно приравнять к $(5-11) \cdot 10^{-4} \%$ по массе.

\section{Л И Т Е Р А Т У Р А}

1. Методические основы исследования химического состава горных пород, руд и минералов. М., 1979, с. 283.

2. В а й н форд н е р Дж. Спектроскопические методы определения следов элементов. М., 1979 , с. 369 .

3. Рентгеноспектральный анализ горных пород с учетом эффекта матрицы по некогерентно рассеянному излучению. Л., 1979.

4. Д о эр фе ль К. Статистика в аналитической химии. М., 1969.
Институт химии
Академии наук Әстонской ССР
Поступила в редакцию
2/VI 1981

\section{R. JUGA, O. HALDNA \\ USALDUSPIIRIDE HINDAMISE METOODIKA MIKROKOMPONENDI MÄARAMISEL RÖNTGENFLUORESTSENTSIMEETODIL}

Mikrokomponendi määramisel röntgenfluorestsentsimeetodil, kusjuures maatriksi mōju arvestatakse proovil mittekoherentselt hajunud kiirguse järgi, sôltub analüüsi tulemuse usalduspiir mikrokomponendi kontsentratsioonist $C$ ja hajunud kiirguse intensiivsusest $I_{\mathrm{H}}$. Esitatud metoodika järgi on võimalik konstrueerida graafikute parv $E_{c}=$ $=\left.F(C)\right|_{{ }_{\mathbf{H}}}$. Nende graafikute alusel saab hinnata mikrokomponendi minimaalset kontsentratsiooni, mille määramine jääb etteantud usalduspiiridesse.

\section{R. JUGA, O. HALDNA}

\section{ESTIMATION OF CONFIDENCE LIMITS FOR THE DETERMINATION OF A MICROCOMPONENT BY X-RAY FLUORESCENCE SPECTROSCOPY}

It has been shown that the confidence limits for the determination of a microcomponent by the X-ray fluorescence spectroscopy with reference to the matrix effect by means of the intensity of noncoherently scattered radiation depend on two factors. They are the concentration of the microcomponent $C$ and the intensity of the radiation $I_{\mathrm{H}}$ scattered on the sample. The method proposed allows us to construct a set of plots $E_{c}=\left.F(C)\right|_{I_{\mathrm{H}}}$ which may be used for the estimation of confidence limits for the determination of $C$. 